\title{
Design of a sunscreen considering the valorization of agroindustrial waste from the wine industry
}

Carrillo-Quezada, Melina ${ }^{1}$, Pariona-La Torre, Luz1, Cárdenas-Toro, Fiorella, $\mathrm{PhD}^{1}$, and Nakama-Hokamura, Keiko, $\mathrm{MSc}^{1}$.

${ }^{1}$ Pontificia Universidad Católica del Perú, Departamento de Ciencias e Ingeniería, Sección de Ingeniería Industrial, melina.carrilloq@pucp.pe, luzk.pariona@pucp.pe, fcardenas@pucp.pe, nakama.gk@pucp.edu.pe

Abstract -- In the present work, a design of a cosmetic product, a sunscreen SPF 50+, was proposed using ingredients obtained from wine industry residues, such as, oil from the grape seed and extract from the grape skin. . The product design begins with the study of the consumer profile, brand preference in the market, attributes most valued in the sunscreen and preference of product size. Then, from this information, a sunscreen is designed that meets the characteristics desired by the consumer, which includes the development of a standard formulation, the physicochemical characteristics and material balance for an estimated pilot production of $100 \mathrm{~kg}$.

Keywords - product design, sunscreen, wine industry residues.

Digital Object Identifier (DOI):

http://dx.doi.org/10.18687/LACCEI2019.1.1.369

ISBN: 978-0-9993443-6-1 ISSN: 2414-6390

$17^{\text {th }}$ LACCEI International Multi-Conference for Engineering, Education, and Technology: "Industry, Innovation, And Infrastructure for Sustainable Cities and Communities", 24-26 July 2019, Jamaica. 


\title{
Design of a sunscreen considering the valorization of agroindustrial waste from the wine industry
}

\author{
Carrillo-Quezada, Melina ${ }^{1}$, Pariona-La Torre, Luz ${ }^{1}$, Cárdenas-Toro, Fiorella, $\mathrm{PhD}^{1}$, and Nakama-Hokamura, Keiko, \\ $\mathrm{MSc}^{1}$.
}

${ }^{1}$ Pontificia Universidad Católica del Perú, Departamento de Ciencias e Ingeniería, Sección de Ingeniería Industrial, melina.carrilloq@pucp.pe, luzk.pariona@pucp.pe, fcardenas@pucp.pe, nakama.gk@pucp.edu.pe

\begin{abstract}
In the present work, a design of a cosmetic product, a sunscreen SPF 50+, was proposed using ingredients obtained from wine industry residues, such as, oil from the grape seed and extract from the grape skin. . The product design begins with the study of the consumer profile, brand preference in the market, attributes most valued in the sunscreen and preference of product size. Then, from this information, a sunscreen is designed that meets the characteristics desired by the consumer, which includes the development of a standard formulation, the physicochemical characteristics and material balance for an estimated pilot production of $100 \mathrm{~kg}$.
\end{abstract}

Keywords: product design, sunscreen, wine industry residues.

Resumen - En el presente trabajo, se realizó una propuesta de diseño de un producto cosmético, un protector solar FPS 50+, a partir de insumos provenientes de los residuos agroindustriales de la industria vitivinícola, aceite de la pepa de uva y extracto de la cáscara de uva. El diseño del producto inicia con el estudio del perfil del consumidor, preferencia de marcas en el mercado, atributos más valorados en el protector solar y preferencia de tamaño del producto. Luego a partir de esta información, se diseña un protector solar que cumpla con las características deseadas por el consumidor, incluyendo el desarrollo de una formulación tipo, características fisicoquímicas y balance de materiales para una producción piloto estimada de $100 \mathrm{~kg}$.

Palabras clave: diseño de producto, protector solar, residuos de la industria vitivinícola

\section{INTRODUCCIÓN}

Mediciones realizadas por la consultora Kantar Worldpanel (2016)[1], muestran que el consumo de productos de cuidado personal registró un crecimiento de $6.3 \%$ en unidades en los hogares peruanos, donde los bloqueadores solares muestran un crecimiento del $26 \%$. Asimismo, se ha incrementado la "cultura de protección", haciendo que 4 de cada 10 hogares peruanos adquieran este tipo de productos. Según una encuesta realizada por el Comité Peruano de Cosmética e higiene (2015)[2] el 96 \% de encuestados afirmó que remplazarían sus productos cosméticos y de higiene personal tradicionales por los orgánicos y naturales.

Digital Object Identifier (DOI):

http://dx.doi.org/10.18687/LACCEI2019.1.1.369

ISBN: 978-0-9993443-6-1 ISSN: 2414-6390
Según datos de la Organización Internacional del Vino (OIV)[3], 100 kilos de uva generan unos 25 kilos de desechos, de los que el $50 \%$ son pieles de uva, el $25 \%$ tallos y el $25 \%$ restante semillas. En la actualidad existen procesos en donde se extrae de la semilla de la uva, antioxidantes[4], que benefician a la piel.

\section{ESTUDIO DEL PERFIL DEL CONSUMIDOR}

El perfil del consumidor, se determinó a través de los resultados obtenidos de la encuesta realizada a 273 personas, muestra del mercado potencial de Lima Metropolitana. Las cuales cumplen con las características definidas en la segmentación del mercado.

\section{- Cálculo del número de muestra:}

La población conformada por el mercado potencial se determinó en la segmentación de mercado, en la cual se considera el número de personas de Lima Metropolitana y el porcentaje que conforma los niveles socioeconómicos a los cuales se centra la empresa que son los niveles A y B, datos obtenidos del APEIM.

También se tomará en cuenta el rango de edad de 25 a 70 años de las personas que se encuentran en los niveles socioeconómicos A y B, porcentajes que al ser multiplicados por el total de personas que conforman Lima Metropolitana, permitirá calcular el número de personas que conforman el mercado potencial que son 1,941,269.

Para poder realizar la encuesta se diseñó un plan de muestreo que aproxime adecuadamente los resultados a la realidad. Por lo tanto, ya que la población es mayor a 100,000 se considera como población infinita y se utilizó la siguiente ecuación (1)[5]:

$$
n=\frac{Z^{2} \times P \times Q}{E^{2}}
$$

Donde:

$\mathrm{n}=$ Número de elementos de la muestra.

$\mathrm{P}=$ Probabilidad de éxito, que para este caso se consideró de $50 \%$.

$\mathrm{Q}=$ Probabilidad de fracaso, que vendría a ser $50 \%$.

$17^{\text {th }}$ LACCEI International Multi-Conference for Engineering, Education, and Technology: "Industry, Innovation, And Infrastructure for Sustainable Cities and Communities", 24-26 July 2019, Jamaica. 
$\mathrm{Z}^{2}=$ Para esta investigación se asumió un nivel de confianza del $90 \%$, por lo tanto, el valor crítico $\mathrm{Z}$ es de 1.64

$\mathrm{E}=$ Margen de error permitido determinado por el equipo de investigación es de $5 \%$.

Reemplazando estos datos en la fórmula se halló que $\mathrm{n}=268.96$, por lo que para obtener información representativa de la población objetivo se deberá encuestar a por lo menos 269 personas.

La encuesta fue realizada entre el 4 y 17 de noviembre del 2017 (Anexo I).

Los aspectos analizados con los resultados de la encuesta son los siguientes:

\section{- Frecuencia de compra de la marca:}

Según las encuestas realizadas, la frecuencia de compra de los protectores solares por parte de las personas es mayor en los meses de verano (Diciembre - Marzo) como se puede observar en la Fig.1. En cuanto al periodo de compra, la tendencia de compra mensual presenta un mejor desempeño, seguido de la tendencia bimensual y quincenal.

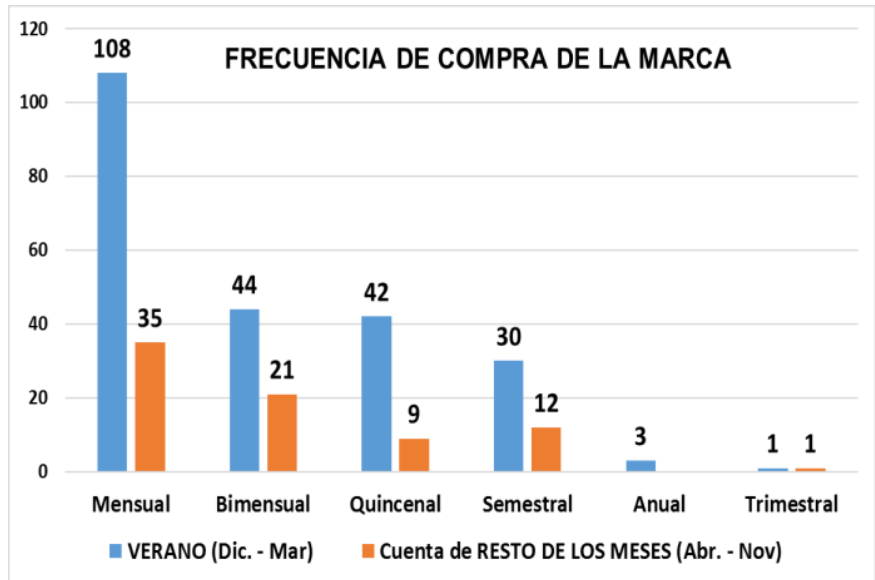

Fig. 1. Frecuencia de compra de la marca

\section{-Preferencia de la marca:}

En cuanto a las marcas de preferencia de las personas en cuanto a protectores solares, se determinó que la marca de mayor aceptación es Unique (31.37\%). Unique es una empresa peruana que es líder en el mercado cosmético, se encuentra presente en nueve países de América y Europa. Cuenta con productos como fragancias, maquillaje, tratamiento facial, bijouterie y productos de cuidado personal.

Luego le sigue la marca de Nivea (15.33\%) y Natura (13.92\%). A continuación, en la Fig.2 de muestran los porcentajes de preferencia de cada una de las marcas:

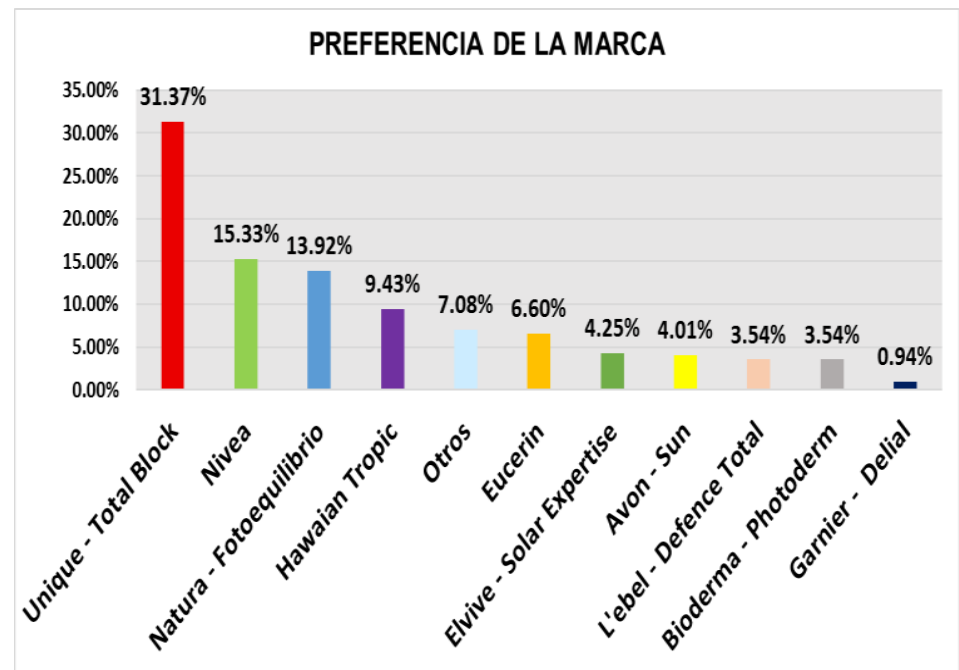

Fig. 2. Preferencia de la marca

\section{- Atributos más valorados de un protector solar:}

En cuanto a los atributos más valorados por las personas (ver Fig. 3) cuando compran un protector solar, se tiene que el factor de protección es el factor de mayor valoración con un $15.90 \%$, seguido del cumplimiento de los beneficios que se muestran en los anuncios o etiquetas con un $14.84 \%$, la calidad de los insumos que usan en sus procesos con un $14.54 \%$. Asimismo, en el caso de los factores como poca sensación de grasosidad en la piel, que el producto se encuentre disponible en los distintos lugares a donde una persona acude, practicidad del envase y el precio, son factores que tienen un porcentaje promedio de $13.68 \%$. Lo cual indica que también son considerados factores relevantes al momento de decidir la compra un protector solar.

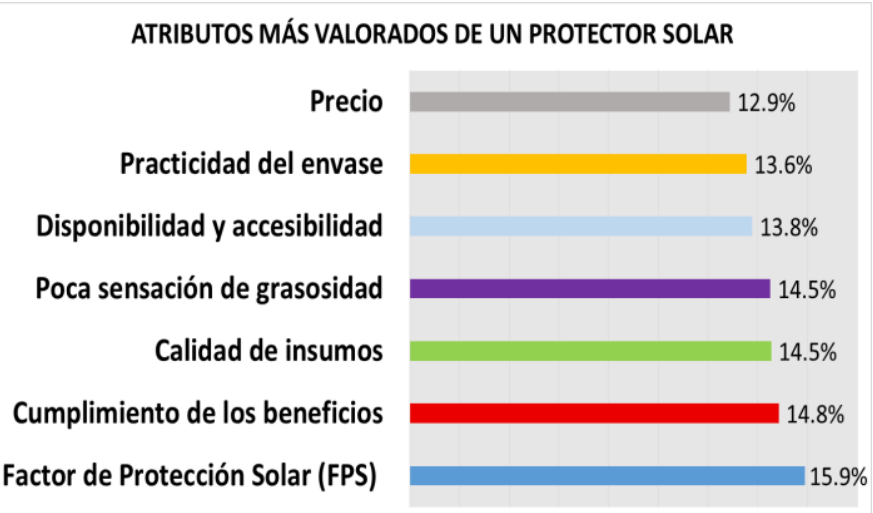

Fig. 3. Atributos más valorados en un protector solar

\section{- Motivo de decisión de compra:}

Con lo que respecta a los motivos por los cuales las personas compran sus marcas preferidas, se pudo determinar que principalmente ello se debe a la Calidad del producto

$17^{\text {th }}$ LACCEI International Multi-Conference for Engineering, Education, and Technology: "Industry, Innovation, And Infrastructure for Sustainable Cities and Communities", 24-26 July 2019, Jamaica. 
(43\%), seguido de la recomendación de conocidos con un $26 \%$ y la recomendación de expertos con un porcentaje de aceptación del $15 \%$.

\section{Finalidad de uso de un protector solar:}

De los resultados obtenidos en la encuesta se puede determinar que las personas utilizan un protector solar principalmente para protegerse de los rayos UVA/UVB (38\%) y un $28 \%$ indica que lo usan para evitar manchas en el rostro.

De esta manera se puede decir que los consumidores de protectores solares son personas preocupadas por el cuidado de la piel de los efectos nocivos de los rayos solares que se van incrementando cada vez más en Perú, por ejemplo, el verano pasado se registraron los valores de radiación más altos del mundo de acuerdo con el Servicio Nacional de Meteorología e Hidrología (SENAMHI) [6]. Los cuales también provocan manchas en la piel, que es la segunda razón más por la que las personas utilizan un protector solar.

\section{- Formas de uso del producto:}

Las personas que utilizan protectores solares prefieren utilizar el producto tanto en el rostro como en el cuerpo, representando el $82 \%$ del total de personas encuestadas. Esto indica que los consumidores no diferencian mucho entre los protectores especializados para el rostro o el cuerpo.

\section{- Lugar de compra:}

En la Fig. 4, se observa la preferencia del lugar de compra de protectores solares, en la encuesta se utilizó una a escala del 1 al 5, siendo 1 nunca, 4 casi siempre y 5 siempre, de esta manera se obtuvo que el $73 \%$ de los encuestados casi siempre y siempre prefiere comprar un bloqueador solar mediante catálogo o consultora, mientras que el $53 \%$ en catálogo y/o consultora. Por otro lado, los lugares donde nunca comprarían un protector solar serían en bodegas y mercados (65\%), centros naturistas $(56 \%)$ y tiendas por departamento $(45 \%)$.

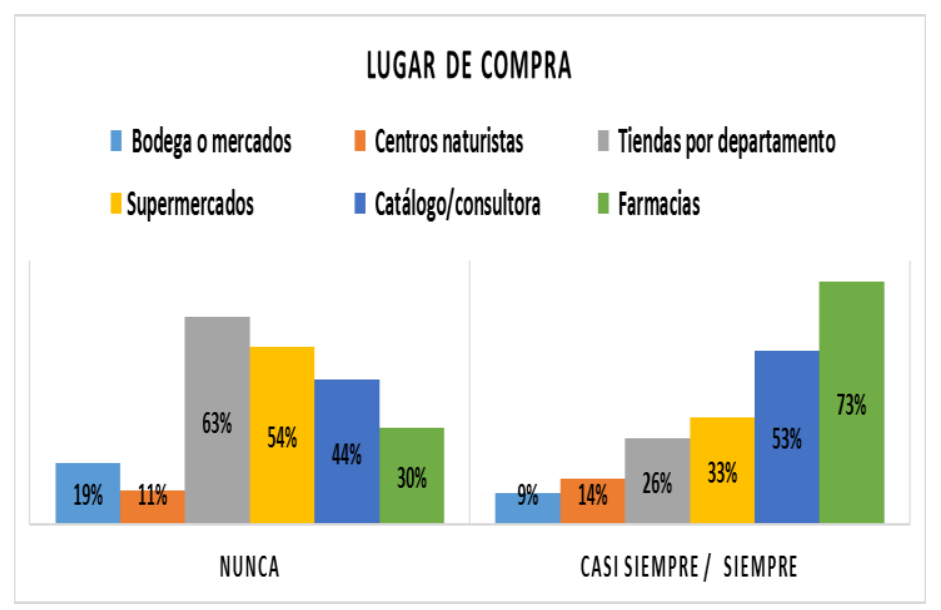

Fig. 4. Lugar de compra

\section{- Utilización del producto:}

Del total de personas encuestadas el 53\% utiliza bloqueadores solares de manera personal y el $47 \%$ restante prefiere utilizarlo de manera familiar.

\section{- Tamaño del producto:}

La presentación del producto será de 90 gr., lo cual también va de acuerdo con la preferencia de los consumidores (ver Fig.5), pues según los resultados de la encuesta el 29\% prefiere una presentación que contenga igual o más de $100 \mathrm{gr}$, el $19 \%$ que contenga 90 gr y el $10 \% 80$ gr que en total hacen el $68 \%$ de los encuestados, razón por la cual se toma el tamaño promedio.

\section{PREFERENCIA DEL TAMAÑO PARA EL PROTECTOR}

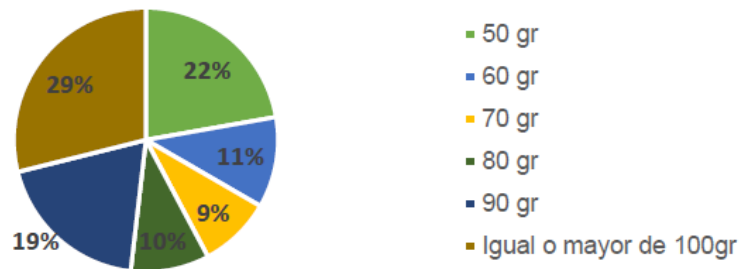

Fig. 5. Preferencia de tamaño para el protector

\section{Estudio DE PRODUCTOS EXISTENTES EN EL MERCADO}

Las principales marcas de bloqueadores solares que lideran el mercado según estudios realizados por CPI [7], son las mostradas en la Figura 6.

RESUMEN COMPARATIVO LÍNEA : BRONCEADORES Y BLOQUEADORES

\begin{tabular}{|l|c|c|c|c|c|}
\hline \multicolumn{1}{|c|}{ MARCAS } & $\begin{array}{c}\text { TOP OF } \\
\text { MIND DE } \\
\text { MARCAS }\end{array}$ & $\begin{array}{c}\text { RECORDACION } \\
\text { ESPONTANEA } \\
\text { TOTAL }\end{array}$ & $\begin{array}{c}\text { MARCA QUE } \\
\text { ACOSTUMBRA } \\
\text { USAR }\end{array}$ & $\begin{array}{c}\text { RECORDACION } \\
\text { PUBLICITARIA } \\
\text { TOTAL }\end{array}$ & $\begin{array}{c}\text { IMAGEN DE } \\
\text { MARCA: } \\
\text { MEIOR } \\
\text { MARCA }\end{array}$ \\
\hline UNIQUE & $\%$ & $\%$ & $\%$ & $\%$ & $\%$ \\
NIVEA & 7.1 & 12.0 & $\mathbf{6 . 2}$ & 5.1 & 6.8 \\
EBEL & 6.1 & 10.2 & $\mathbf{3 . 8}$ & 2.5 & 4.2 \\
HAWAIAN TROPI & 4.8 & 8.4 & $\mathbf{3 . 6}$ & 2.3 & 2.6 \\
AVON & 4.6 & 7.7 & 4.3 & 1.4 & 3.6 \\
& 3.0 & 5.5 & $\mathbf{2 . 3}$ & 1.0 & 1.4 \\
\hline
\end{tabular}

Target: Personas de 14 a 64 años del ABCDE

Muestra: 1000

PERIODO: AGOSTO 2008

FUENTE: COMPAÑÍA PERUANA DE ESTUDIOS DE MERCADO Y OPINIÓN PÚBLICA SAC.

Fig. 6. Marcas de bloqueadores preferidas por los consumidores de Lima

$17^{\text {th }}$ LACCEI International Multi-Conference for Engineering, Education, and Technology: "Industry, Innovation, And Infrastructure for Sustainable Cities and Communities", 24-26 July 2019, Jamaica. 
También se encuentran marcas como Garnier, Bioderma, Unique, Elvive, Bahía, Banana Boat, entre otros. Por lo tanto, el número de empresas que cuentan con marcas de protectores solares en el Perú y que se encuentren bien posicionadas en el sector, no es muy grande.

De las marcas con mayor porcentaje de reconocimiento por parte de la población encestada se realizaron los siguientes cuadros comparativos de sus principales características de modo que se puede observar sus semejanzas y los factores de diferenciación entre unos y otros.

TABLA I

CUADRO COMPARATIVO DE LOS PRINCIPALES PRODUCTOS

\begin{tabular}{|c|c|c|c|}
\hline Empresa & $\begin{array}{l}\text { Variedad de } \\
\text { presentaciones }\end{array}$ & Precio & $\begin{array}{l}\text { Factor de } \\
\text { protección } \\
\text { solar }\end{array}$ \\
\hline $\begin{array}{l}\text { Unique - } \\
\text { Total Block }\end{array}$ & $\begin{array}{l}\text { Presentación en Frasco } 80 \text { gr } \\
\text { (UVB SPF 100) }\end{array}$ & S/. 59.00 & $\begin{array}{c}\text { SPF } 100,70 y \\
50+\end{array}$ \\
\hline \multirow{3}{*}{ Nivea } & $\begin{array}{l}\text { Presentación en Frasco } 200 \\
\text { ml (Nivea Sun Invisible } \\
\text { Protection Spray) }\end{array}$ & S/. 49.90 & SPF 50 \\
\hline & $\begin{array}{l}\text { Presentación en Frasco } 125 \\
\text { ml (Nivea Sun Hidrata) }\end{array}$ & S/. 22.90 & SPF 50 \\
\hline & $\begin{array}{l}\text { Presentación en Spray } 200 \\
\text { ml (Nivea Sun } \\
\text { Protect\&Refresh) }\end{array}$ & S/. 44.17 & SPF 50 \\
\hline $\begin{array}{l}\text { Natura - } \\
\text { Fotoequilibr } \\
\quad \text { io }\end{array}$ & $\begin{array}{l}\text { Presentación en Frasco } 120 \\
\text { ml (Protector solar para el } \\
\text { cuerpo) }\end{array}$ & S/. 55.00 & SPF 30 y 50 \\
\hline \multirow{2}{*}{$\begin{array}{l}\text { Hawaian } \\
\text { Tropic }\end{array}$} & $\begin{array}{l}\text { Presentación en Frasco } 240 \\
\text { ml (Hawaiian Tropic Sheer } \\
\text { SPF30) }\end{array}$ & S/. 29.90 & SPF 30 \\
\hline & $\begin{array}{l}\text { Presentación en Spray } 170 \mathrm{gr} \\
\text { (Hawaiian Tropic Island } \\
\text { Sport) }\end{array}$ & S/. 56.90 & SPF 50 \\
\hline $\begin{array}{l}\text { Producto } \\
\text { propuesto }\end{array}$ & Presentación en Frasco 90 gr & $\mathrm{S} / .85 .00$ & SPF 50+ \\
\hline
\end{tabular}

TABLA II

CUADRO COMPARATIVO DE LOS PRINCIPALES PRODUCTOS

\begin{tabular}{|c|c|c|c|}
\hline \multirow{2}{*}{ Empresa } & $\begin{array}{c}\text { Uso de productos } \\
\text { naturales }\end{array}$ & Certificaciones & $\begin{array}{c}\text { Posicionamient } \\
\text { o en el } \\
\text { mercado* }\end{array}$ \\
\hline $\begin{array}{c}\text { Unique }- \\
\text { Total Block }\end{array}$ & No & Sí & $19.4 \%$ \\
\hline \multirow{2}{*}{ Nivea } & No & No & \multirow{2}{*}{$15.3 \%$} \\
\cline { 2 - 3 } & No & No & \multirow{2}{*}{$6.8 \%$} \\
\cline { 2 - 3 } $\begin{array}{c}\text { Natura - } \\
\text { Fotoequilibri } \\
\text { o }\end{array}$ & $\begin{array}{c}\text { Sín (tomate y semilla } \\
\text { de café verde) }\end{array}$ & Sí & \multirow{2}{*}{$0.8 \%$} \\
\hline \multirow{2}{*}{$\begin{array}{c}\text { Hawaian } \\
\text { Tropic }\end{array}$} & $\begin{array}{c}\text { Sí (ingredientes } \\
\text { tropicales) }\end{array}$ & $\begin{array}{c}\text { Sí (ingredientes } \\
\text { tropicales) }\end{array}$ & Sí \\
\cline { 2 - 3 } & Sí & \\
\hline $\begin{array}{c}\text { Producto } \\
\text { propuesto }\end{array}$ & $\begin{array}{c}\text { Sí (aceite y extracto } \\
\text { de uva) }\end{array}$ & Sí (deseable) & \multicolumn{1}{|c|}{-} \\
\hline
\end{tabular}

* Según el estudio de kantar worldpanel julio 2017 - junio 2018

Por otro lado, las empresas con grandes volúmenes de ventas y que son especializadas en la venta de productos farmacéuticos, dentro de los cuales se encuentran los protectores solares, tienen ventajas absolutas en costos, entre los que destaca los altos costos fijos, grandes requerimientos de capital, alta inversión en $\mathrm{I}+\mathrm{D}$ y publicidad destinada a generar lealtad en los consumidores.

De acuerdo con los resultados de la Encuesta Nacional de Innovación en la Industria Manufacturera [8] 2015 el 66\% de las empresas que fabrican productos farmacéuticos en Perú invierten en algún tipo de innovación, esto hace que los productos ya existentes en el mercado sufran cambios y mejoras cada periodo de tiempo creando una fuerte competencia entre estos productos por abarcar un mayor porcentaje del mercado.

El bajo número de competidores, junto al crecimiento del consumo de bloqueadores en el Perú [9], hace que la rivalidad entre competidores pueda parecer baja. Sin embargo, la inversión en costos fijos, capital, en I+D y publicidad necesarios para mantenerse y posicionarse en el mercado hace que la rivalidad entre competidores sea alta, pues una pequeña empresa tendría muchas dificultades para asumir estos costos.

\section{DISEÑO DE PRODUCTO}

Teniendo en consideración lo señalado en la primera parte del estudio, se propone una formulación del protector solar a partir de insumos obtenidos de la semilla y cáscara de uva: aceite de la semilla de uva y extracto concentrado en resveratrol.

La producción del aceite puede ser llevado a cabo utilizando diferentes técnicas; siendo las más usadas el prensado en frío, extracción por soxhlet y la tecnología de fluidos supercríticos. De la misma forma, la obtención de un extracto enriquecido con resveratrol puede ser obtenido por diferentes técnicas como la maceración con solventes acuosos y orgánicos, así como el uso de la tecnología de fluidos presurizados. Existen estudios que indican que el uso del aceite de uva y el extracto de uva confiere propiedades antioxidantes; así como el aporte de propiedades emolientes en la textura del producto cosmético.

Teniendo en consideración la demanda por productos obtenidos a partir de tecnologías verdes que tengan un menor impacto al medio ambiente y el uso de solventes GRAS (Generally Recognized as Safe), surge la importancia de proponer nuevos desarrollos utilizando tecnologías a altas presiones o tecnología por fluidos supercríticos. En la Figura 7, se muestra un esquema de la obtención del aceite usando como solvente el dióxido de carbono a partir de la tecnología por fluidos supercríticos. La extracción con fluidos supercríticos es una operación unitaria que consiste en la separación de un compuesto de interés (soluto) presente en una matriz sólida a través del contacto con un disolvente supercrítico. El disolvente más ampliamente utilizado en la industria alimentaria

$17^{\text {th }}$ LACCEI International Multi-Conference for Engineering, Education, and Technology: "Industry, Innovation, And Infrastructure for Sustainable Cities and Communities”, 24-26 July 2019, Jamaica. 
es el dióxido de carbono ( $\mathrm{CO} 2)$ debido a su temperatura y presión crítica moderada, disponibilidad en alta pureza y aprobación en el procesamiento de alimentos. Este solvente disuelve componentes apolares y poco polares; presenta una baja solubilidad para pigmentos y agua y no se disuelve proteínas, polisacáridos, azúcares y minerales [10]. Debido a la baja polaridad del dióxido de carbono, modificadores (compuesto de alta polaridad) o co-disolventes se añaden al proceso en pequeñas cantidades, con la que se puede aumentar la cantidad de compuestos a ser extraídos $[11,12]$

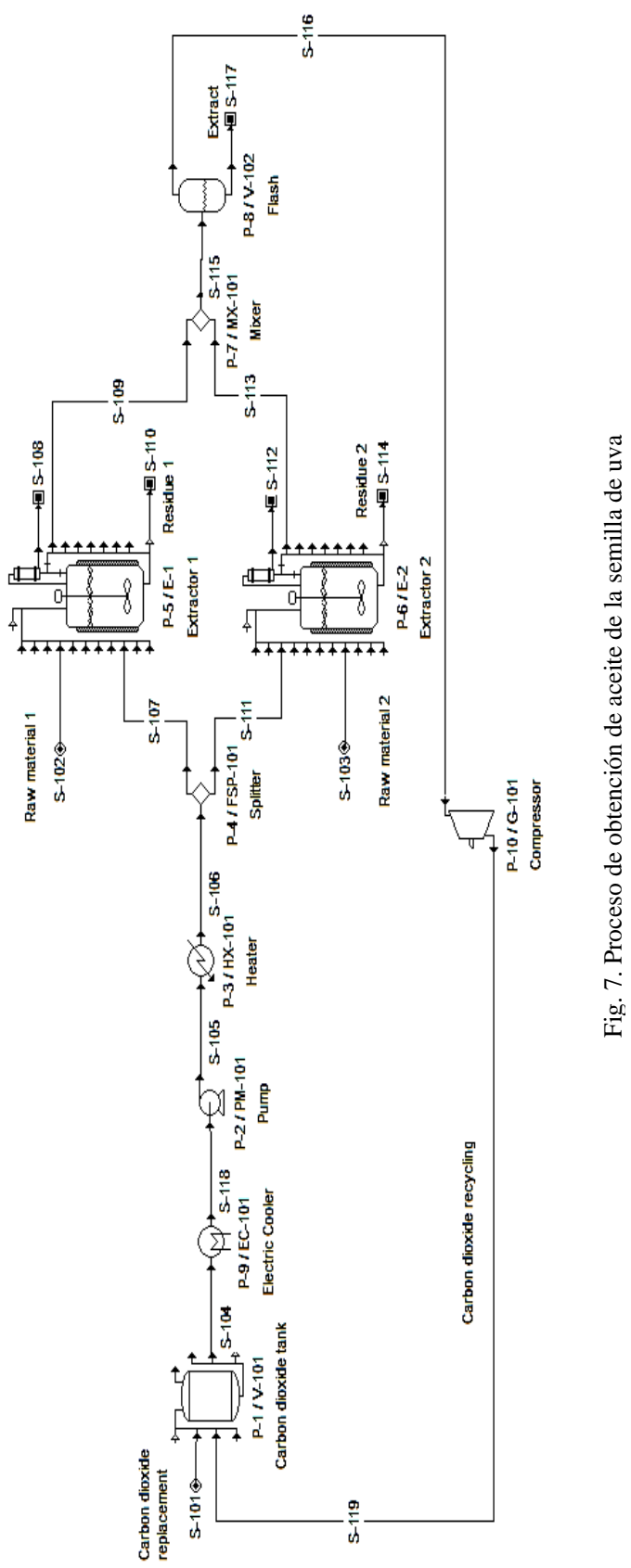

Desarrollo y resultados de la formulación química

El desarrollo del protector solar toma en consideración en las siguientes fases: (1) Diseño de fórmula conforme a una referencia comercial, en este caso Unique Total Block, con protección solar SPF50+, presentación en frasco de $90 \mathrm{~g}$ (2) Desarrollo de 3 prototipos con aceite y extractos a escala piloto para evaluación de estabilidad del producto, el cual incluye las propiedades fisicoquímicas del producto $(\mathrm{pH}$, viscosidad, estabilidad de la emulsión) y análisis microbiológico a las condiciones de $0^{\circ} \mathrm{C}, 40^{\circ} \mathrm{C}$ y $25^{\circ} \mathrm{C}$ por un periodo de 3

$17^{\text {th }}$ LACCEI International Multi-Conference for Engineering, Education, and Technology: "Industry, Innovation, And Infrastructure for Sustainable Cities and Communities", 24-26 July 2019, Jamaica. 
meses, según lo recomendado por la autoridad sanitaria del Perú (DIGEMID).

En la industria cosmética es válido utilizar un modelo teórico para la predicción del factor de protección solar (SPF). El software BASF Sunscreen Simulator ${ }^{\circledR}$ predice con un alto grado de confiabilidad del SPF teórico respecto al SPF in vivo [13]. En el presente trabajo, se ha considerado el siguiente desarrollo de fórmula SPF 50+:

\begin{tabular}{|c|c|c|}
\hline $\mathrm{N}^{\circ}$ & INCI Name & $\%$ \\
\hline 1 & Water & 53.50 \\
\hline 2 & Octyl Methoxycinnamate & 10.00 \\
\hline 3 & Octocrylene & 7.00 \\
\hline 4 & Diethyl Hexylbutamido triazona & 6.00 \\
\hline 5 & $\begin{array}{l}\text { Cetearyl Alcohol (and) Glyceryl stearate (and) PEG-40 stearate (and) } \\
\text { Ceteareth-20 }\end{array}$ & 4.50 \\
\hline 6 & Diisobutyl Adipate & 3.50 \\
\hline 7 & $\begin{array}{l}\text { Bis-Ethylhexyloxyphenol } \\
\text { Methoxyphenyl Triazine }\end{array}$ & 4.00 \\
\hline 8 & C12-15 Alkyl Benzoate & 2.50 \\
\hline 9 & $\begin{array}{l}\text { Glyceryl stearate (and) PEG-100 } \\
\text { Stearate }\end{array}$ & 2.50 \\
\hline 10 & Vitis Vinifera Seed Oil & 2.50 \\
\hline 11 & Glycerin & 2.00 \\
\hline 12 & Ethylhexylglycerin (and) Phenoxyethanol & 0.60 \\
\hline 13 & Vitis Vinifera Fruit Extract & 0.60 \\
\hline 14 & Polyquaternium 37 & 0.50 \\
\hline 15 & Fragance & 0.30 \\
\hline & Total & 100.00 \\
\hline
\end{tabular}

Los resultados del simulador indican un valor teórico de SPF de 56.9, UVA-PF de 7.2 in vitro (ISO 24443) y UVA-PF de 9.4 in vitro (ISO 24442). Respecto a las propiedades fisicoquímicas del protector solar, en base a ensayos de laboratorio, se encuentran en el siguiente rango:

- pH: $4.0-6.0$

- Viscosidad: 200,000 y 400,000 cps

- Prueba centrífuga: Sin separación de fases.

- Pruebas microbiológicas: Recuento total de microorganismos aerobios $<10 \mathrm{UFC} / \mathrm{g}$, Recuento total combinado de hongos filamentosos y levaduras $<10$ UFC/g, Pseudomonas auregionsa y Staphylococcus aureus en $1 \mathrm{~g}$, ausente.

\section{Procedimiento operacional piloto propuesto:}

En un tanque se mezclan los ingredientes solubles en fase oleosa: (a)adicionar los insumos (6), (8), (2), (3), (4), (7), (5) y

(9) y calentar a temperatura menor de $75^{\circ} \mathrm{C}$ y homogenizar hasta obtener una mezcla líquida translúcida sin grumos. En otro tanque se mezclan los ingredientes solubles en fase acuosa: (a) adicionar los insumos $95 \%$ de (1), (11) y (14) y homogenizar a temperatura ambiente hasta obtener una mezcla gelificada sin grumos. En el tanque que contiene la fase acuosa se adiciona la fase oleosa a temperatura menor de $75^{\circ} \mathrm{C}$ y se realiza la emulsión. Realizar enfriamiento lentamente con agitación constante hasta temperatura menor a $40^{\circ} \mathrm{C}$. Adicionar el insumo (15) con agitación lenta y enfriar hasta temperatura

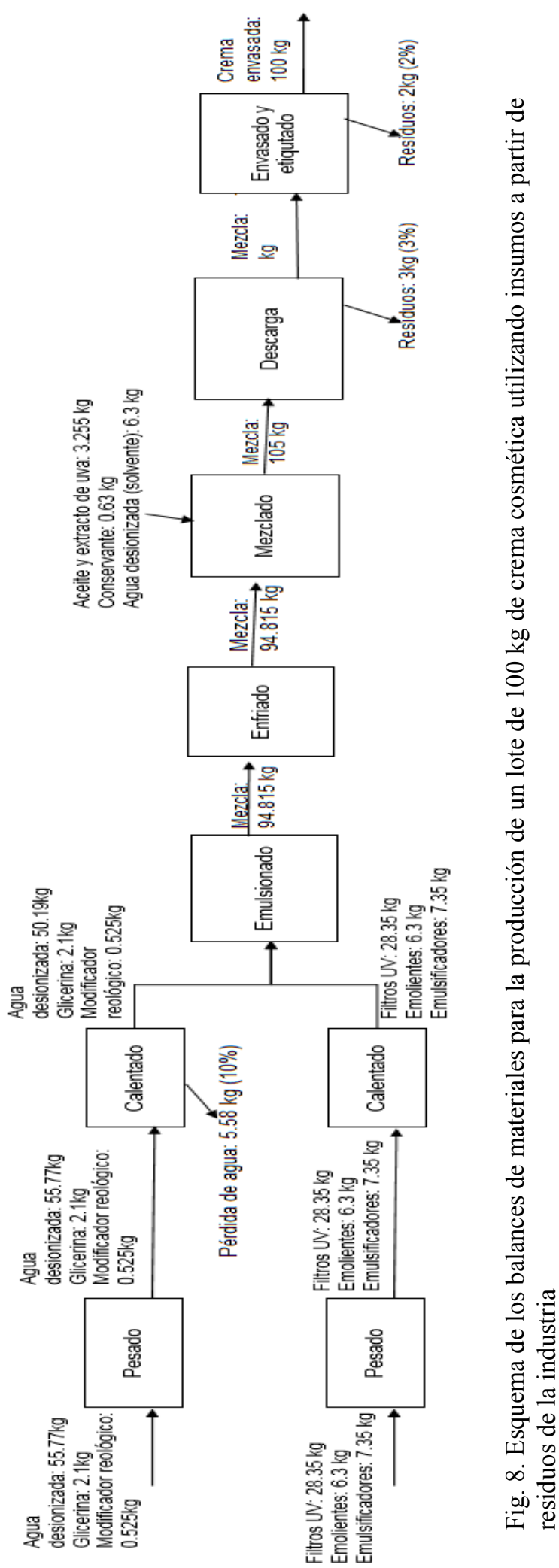

$17^{\text {th }}$ LACCEI International Multi-Conference for Engineering, Education, and Technology: "Industry, Innovation, And Infrastructure for Sustainable Cities and Communities", 24-26 July 2019, Jamaica. 
ambiente. Medir $\mathrm{pH}$, viscosidad y prueba con centrífuga para verificar la ausencia de separación de fases en la emulsión.

La Fig. 8 muestra los requerimientos de materiales para la producción de un batch de $100 \mathrm{~kg}$ de protector solar, el cual es el tamaño mínimo estándar de los maquiladores de productos cosméticos en Perú.

\section{CONCLUSIONES}

En el presente trabajo se presenta una propuesta de diseño de un producto cosmético a partir de insumos obtenidos a partir de los residuos de la agroindustria. La propuesta incluye el estudio de investigación de mercado, el desarrollo teórico del producto, y su escalamiento a nivel piloto - industrial. Esta propuesta ofrece una alternativa de uso a los residuos de origen agroindustrial.

\section{REFERENCIAS}

[1] El Comercio. Consumo de productos de cuidado personal aumentó 6.3\% a Mayo. Consulta 01 de Marzo de 2019. https://elcomercio.pe/economia/negocios/consumo-productos-cuidadopersonal-aumento-6-3-mayo-246928?foto=

[2] El Comercio. Perú apuesta por la cosmética en base a productos naturales. Consulta 01 de Marzo de 2019. https://elcomercio.pe/economia/negocios/consumo-productos-cuidadopersonal-aumento-6-3-mayo-246928?foto=

[3] AINIA. Tres vidas para la uva. Consulta 01 de Marzo de 2019. https://www.ainia.es/noticias/alimentacion-saludable/tres-vidas-para-lauva/

[4] AINIA. Es posible tener más valor de los residuos de procesado de uva. $\begin{array}{llllll}\text { Consulta } & 01 & \text { de } & \text { Marzo de } & \end{array}$ https://www.ainia.es/tecnoalimentalia/formacion/es-posible-obtener-masvalor-de-los-residuos-del-procesado-de-la-uva/

[5] MCDANIEL, Carl / GATES, Roger. 2011. Investigación de mercados. 8a edición. México: Cengage Learning

[6] RPP. Senamhi. El Perú sufrirá la radiación más alta del mundo en febrero. Consulta: 23 de diciembre de 2018.

https://rpp.pe/politica/actualidad/peru-afrontara-en-febrero-radiacion-masalta-del-mundo-advierte-especialista-noticia-1027716

[7] CPI. 2017. Audiencias radiales. Consulta: 25 de mayo de 2018 https://cpi.pe/images/upload/paginaweb/archivo/23/200808_BT_BRONC EADORES_BLOQUEADORES.pdf

[8] ESAN. 2017. La industria de productos farmacéuticos en Perú. Consulta: 10 de enero de 2019.

https://www.esan.edu.pe/apuntes-empresariales/2017/04/la-industria-deproductos-farmaceuticos-en-peru/

[9] BEATYPROF. 2016. El mercado de protectores solares incrementó sus ventas un 7,7\% en 2015. Consulta: 10 de enero de 2019. http://www.revistabeautyprof.com/es/notices/2016/04/el-mercado-deprotectores-solares-incremento-sus-ventas-un-7-7-en-201571919.php\#.XDv7o1wzbIU

[10] BRUNNER, G. Supercritical fluids: technology and application to food processing. Journal of Food Engineering. v. 67, n. 1-2, p. 21-33, 2005.

[11] PASQUEL, A.; MEIRELES, M.A.A.; MARQUES, M.O.M.;PETENATE, A.J. Extraction of stevia glycosides with $\mathrm{CO}_{2}+$ water, $\mathrm{CO}_{2}+$ ethanol, and $\mathrm{CO}_{2}+$ water + ethanol. Brazilian Journal of Chemical Engineering. v.17, n.3, pp. 271-282, 2000.

[12] HERRERO, M.; CIFUENTES, A.; IBAÑEZ, E. Sub- and supercritical fluid extraction of functional ingredients from different natural sources: Plants, food-by-products, algae and microalgae: A review. Food Chemistry. v. 98, n. 1, p. 136-148, 2006.
[13] Santos Caetano, J. P., Abarca, A. P., Guerato, M., Guerra, L., Schalka, S., Perez Simão, D. C., \& Vila, R. (2016). SPF and UVASPF sunscreen evaluation: are there good correlations among results obtained in vivo, in 
Edad:

Distrito:

\section{Género}
Femenino
Masculino

2. Estado Civil
Soltero (a)
Casado (a)
Viudo (a)
Divorciado (a)

3. ¿Cuál es su profesión?

4. ¿Utiliza protectores solares?

$\square$ Sí
$\square$ No

Responder, si la pregunta 4 fue positiva

5. ¿Con qué frecuencia compra el producto?

Considerando una presentación de 50 gr

\begin{tabular}{|l|l|l|}
\hline & $\begin{array}{c}\text { En el verano } \\
\text { (Dic. - Mar) }\end{array}$ & $\begin{array}{c}\text { En el resto de } \\
\text { los meses } \\
\text { (Abr. - Nov) }\end{array}$ \\
\hline Quincenal & & \\
\hline Mensual & & \\
\hline Bimensual & & \\
\hline Semestral & & \\
\hline
\end{tabular}

Responder, si la pregunta 4 fue positiva

6. Según su preferencia, ordene sus tres marcas más importantes, donde 1 es el más importante.

\begin{tabular}{|c|c|c|}
\hline Marca & Imagen & Preferencia \\
\hline Unique - & & \\
Total block & & \\
$(\mathrm{A})$ & & \\
& & \\
\hline Nivea (B) & & \\
\hline L'ebel - & & \\
Défence Total & & \\
$(\mathrm{C})$ & & \\
\hline
\end{tabular}

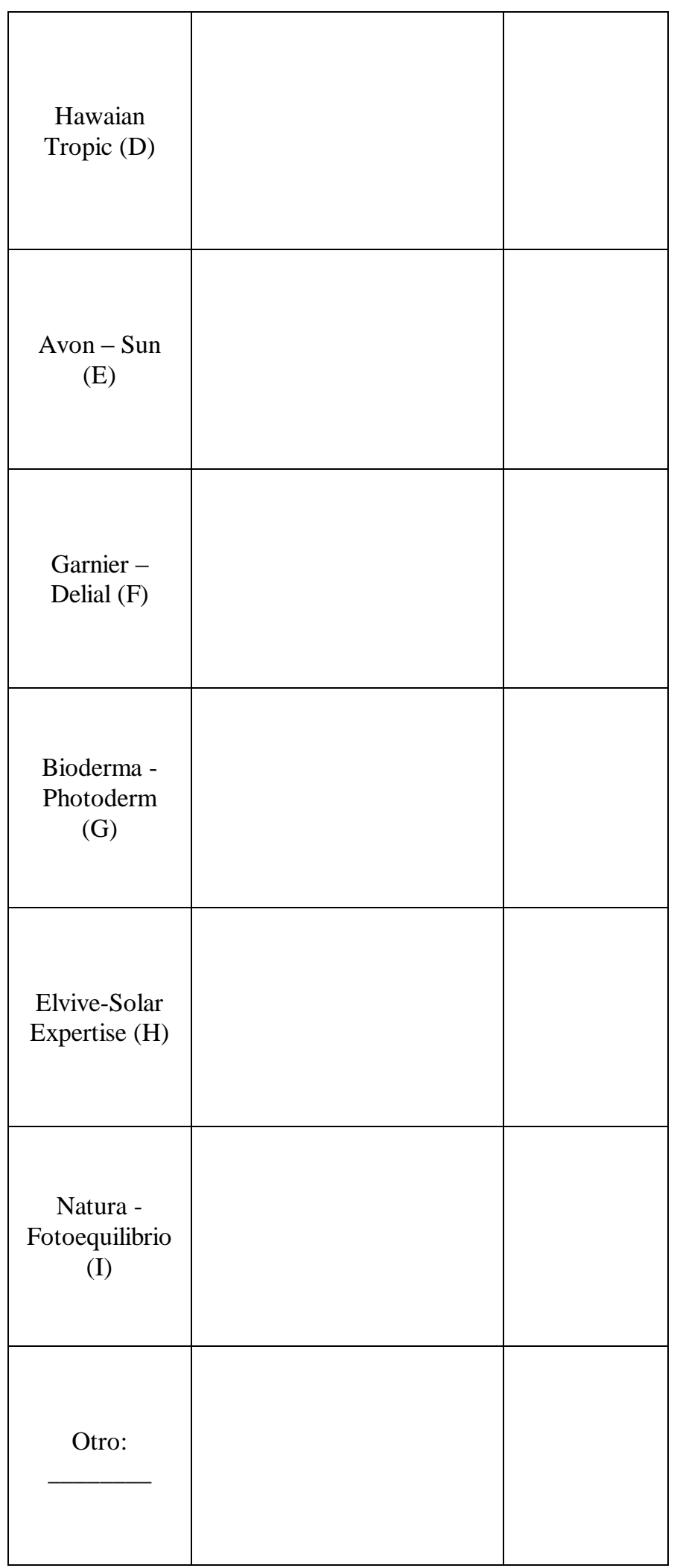

Responder, si la pregunta 4 fue positiva 
7. ¿Qué valora de un protector solar? Donde: 1 es menos importante y 5 es más importante.

\begin{tabular}{|l|l|l|l|l|l|}
\hline & 1 & 2 & 3 & 4 & 5 \\
\hline $\begin{array}{l}\text { Factor de protección solar } \\
\text { (FPS) }\end{array}$ & & & & & \\
\hline Calidad de insumos & & & & & \\
\hline $\begin{array}{l}\text { Cumplimiento de los } \\
\text { beneficios ofrecidos }\end{array}$ & & & & & \\
\hline Precio & & & & & \\
\hline Practicidad del envase & & & & & \\
\hline Disponibilidad y accesibilidad & & & & & \\
\hline $\begin{array}{l}\text { Poca sensación de grasosidad } \\
\text { en la piel }\end{array}$ & & & & & \\
\hline Otros: & & & & & \\
\hline
\end{tabular}

8. ¿Por qué compra su marca preferida?

Precio

Recomendación de conocidos

Recomendación de expertos

Reconocimiento de la marca

Calidad del producto

Presentación

Otros:

Responder, si la pregunta 4 fue negativa

9. ¿Estaría dispuesto a utilizar protectores solares?

$\square$ Sí
$\square$ No

Si la respuesta es sí, continúe con la pregunta 9; caso contrario concluyó la encuesta:

10. ¿Con qué fin utiliza/utilizaría protector solar?

(Puede marcar más de una alternativa)

$\square \quad$ Evitar manchas en la piel

$\square$ Hidratación

Prevenir el envejecimiento

Protección contra los rayos UVA/UVB

Todas las anteriores

Otros:

11. Cuando compra un protector solar, ¿le interesa que solo sea para el rostro o también para el cuerpo?

Solo para el rostro

Solo para el cuerpo

Ambos
12. ¿En qué medio publicitario le gustaría recibir información del producto?

(Puede marcar más de una alternativa)

\section{Facebook}

Twitter

Página web

Tv

Radio

Correo electrónico

Otros:

13. Enumere del 1 al 5, según su preferencia, el lugar de compra del producto. 1=nunca, $2=$ rara vez, $3=a$ veces, 4=normalmente, $5=$ siempre

\begin{tabular}{|l|l|l|l|l|l|}
\hline & 1 & 2 & 3 & 4 & 5 \\
\hline Catálogo/Consultora & & & & & \\
\hline Farmacias & & & & & \\
\hline Supermercado & & & & & \\
\hline Bodega o mercado & & & & & \\
\hline Centros naturistas & & & & & \\
\hline $\begin{array}{l}\text { Tiendas por } \\
\text { departamento }\end{array}$ & & & & & \\
\hline Otros: & & & & & \\
\hline
\end{tabular}

14. ¿Qué tipo de promoción le parece más atractiva?

\section{2x1}

Segundo producto a mitad de precio

$\square$ Pack bloqueador + crema facial humectante

$\square$ Pack bloqueador $90 \mathrm{gr}+$ presentación de bolsillo

\section{Preguntas obligatorias}

15. Estaría dispuesto a comprar un protector solar con aceite de uva, el cual tiene propiedades rejuvenecedoras, antiinflamatorias que ayudan a desobstruir los poros y eliminar el acné, y además tiene la capacidad de retener humedad y mantener la piel tersa y suave, propiedades que un protector común no le brindaría.

$\square \quad$ Sí
$\square \quad$ No

Si la respuesta es sí, continúe con la pregunta 16; caso contrario concluyó la encuesta:

16. De los siguientes nombres. ¿Cuál considera más apropiado para el protector solar propuesto? Donde $1=$ primera opción y $2=$ segunda opción

$17^{\text {th }}$ LACCEI International Multi-Conference for Engineering, Education, and Technology: "Industry, Innovation, And Infrastructure for Sustainable Cities and Communities”, 24-26 July 2019, Jamaica. 


\begin{tabular}{|l|l|}
\hline BioBlock & \\
\hline Natural Block & \\
\hline Active Protect & \\
\hline Photo Block & \\
\hline Photo Protect & \\
\hline
\end{tabular}

17. ¿Utilizaría el protector solar antes mencionado para uso personal o familiar?

$\square$ Uso personal

$\square \quad$ Uso familiar

18. ¿En qué tamaño le gustaría comprar el protector solar? (gr)

$\square \quad 50$

$\square \quad 60$

$\square 70$

$\square 80$

$\square 90$

$\square \quad$ Igual o mayor de 100:

19. ¿Cuánto estaría dispuesto a pagar por un protector solar de $90 \mathrm{gr}$ ?

Menos de S/. 70:

$\square$ S/. 70

$\square$ S/. 80

$\square$ S/. 90

$\square$ S/. 100

Más de S/. 100:

Nombre y Apellido:

Firma:

Muchas gracias por su tiempo y colaboración

$17^{\text {th }}$ LACCEI International Multi-Conference for Engineering, Education, and Technology: "Industry, Innovation, And 